\title{
Contralateral S1 nerve root transfer for motor function recovery in the lower extremity among patients with central nervous system injury: study protocol for a randomized controlled trial
}

\author{
Ying Peng ${ }^{1 \# \wedge}$, Shi-Feng Kan ${ }^{2 \#}$, Wei-Jie Ren ${ }^{3 \#}$, Zhuo-Xuan Li ${ }^{1}$, Gang Yin $^{1}$, Bo Yu ${ }^{2}$, Hao-Dong Lin $^{1} \wedge$ \\ ${ }^{1}$ Trauma Center, Shanghai General Hospital, Shanghai Jiao Tong University School of Medicine, Shanghai, China; ${ }^{2}$ Department of Rehabilitation, \\ Shanghai General Hospital, Shanghai Jiao Tong University School of Medicine, Shanghai, China; ${ }^{3}$ Department of Radiology, Shanghai General \\ Hospital, Shanghai Jiao Tong University School of Medicine, Shanghai, China \\ \#These authors contributed equally to this work. \\ Correspondence to: Bo Yu. Department of Rehabilitation, Shanghai General Hospital, Shanghai Jiao Tong University School of Medicine, 100 Haining \\ Road, Hongkou District, Shanghai 200080, China. Email: boyujtu@163.com; Hao-Dong Lin. Trauma Center, Shanghai General Hospital, Shanghai \\ Jiao Tong University School of Medicine, 100 Haining Road, Hongkou District, Shanghai 200080, China. Email: haodonglin@hotmail.com.
}

\begin{abstract}
Background Central nervous system injury (CNSI) comprises a series of common diseases that severely affect patients' motor function and quality of life and is associated with high disability and mortality rates. Previous studies have shown that contralateral lumbosacral nerve root transfer significantly improved the function of the paralyzed limb in rat models of CNSI. These studies showed that severing the sacral 1 nerve root (S1) did not damage the function of the ipsilateral lower extremity. Thus, we speculate that contralateral S1 nerve root transfer can improve the recovery of a paralyzed limb. Because no associated rigorously designed randomized controlled trial has evaluated the effectiveness of contralateral S1 nerve transfer thus far, we designed this clinical trial to compare the effects of this new treatment approach with those of traditional treatments in paralyzed patients after chronic CNSI.
\end{abstract}

Methods: This is a single-center, prospective, randomized controlled trial. Forty patients, who meet the inclusion criteria and have hemiplegia caused by chronic CNSI, will be randomly divided into the surgical or non-surgical group. The treatment effect in the 2 groups will be assessed before and 3, 6, 9, 12, 18, and 24 months after intervention by using numerous scales and resting-state functional magnetic resonance imaging. The primary outcome will be the Fugl-Meyer score for the lower limbs 24 months after treatment. The secondary outcomes include the modified Ashworth spasm scale, the modified Barthel scale, 10-m walking speed measurement results, three-dimensional gait analysis, muscle strength testing, electromyography, and resting-state functional magnetic resonance imaging findings. Safety outcomes and adverse events will be observed simultaneously.

Discussion: We expect that the surgery will improve the sensorimotor functions of the paralyzed limb, and the results of this trial will provide high-quality clinical evidence for a new efficient treatment strategy for disability after CNSI.

Trial registration: Chinese Clinical Trial Registry: ChiCTR1800014414, registration date: 12 January 2018.

Keywords: Nerve transfer; motor function reconstruction; stroke; traumatic brain injury; randomized controlled trial

Submitted Jan 22, 2021. Accepted for publication May 14, 2021.

doi: $10.21037 / \mathrm{apm}-21-183$

View this article at: http://dx.doi.org/10.21037/apm-21-183

^ ORCID: Ying Peng, 0000-0002-4247-391X; Haodong Lin, 0000-0001-5414-7516. 


\section{Introduction}

Central nervous system injury (CNSI), including stroke, traumatic brain injury (TBI), and cerebral palsy, is one of the significant sets of diseases that seriously endanger human health and life $(1,2)$. It is characterized by multiple complications and high morbidity, disability, and mortality rates $(3,4)$. It affects numerous people worldwide, especially in developing countries $(5,6)$. The World Health Organization (WHO) estimated that 5.7 million deaths and 16 million first-time events were caused by stroke in 2005, and without intervention, these numbers may reach 7.8 million and 23 million, respectively, by 2030 (7). After a stroke, approximately $80 \%$ of patients experience limb motor impairment, and two-thirds of patients with functional defects of the lower limbs cannot walk independently after a stroke (3), which severely affects their daily life and leads to a substantial financial burden to society. Unfortunately, the current treatments for stroke are limited and unsatisfactory. The main treatments for stroke are the prevention of new episodes and acute relief of the cause of stroke, such as thrombolysis or closure of the target artery (8).

Furthermore, treatments for the chronic period of stroke are few and ineffective. Thus, it is of great social and economic significance to find an effective treatment for paralyzed patients in the chronic phase of CNSI. At present, rehabilitation is the main treatment for the chronic phase of CNSI. However, only about $30 \%$ of patients can partly recover their motor function, and the curative effect is unsatisfactory (9). Other therapies such as stem cell therapy are still in their infancy, and understandably, no breakthrough has yet been achieved in the case of stroke treatment $(10,11)$.

Previous studies have shown that a healthy cerebral hemisphere controls the paralyzed limb through a potential ipsilateral peripheral nerve pathway for compensation, vital for limb function recovery after CNSI. However, due to a lack of enough ipsilateral neural pathways and significant variability in anatomy (12), this spontaneous compensation is limited and functional recovery is far from satisfactory (13). Hence, we speculated whether we could artificially create a new neural pathway to ensure the healthy hemisphere controls paralyzed limbs more efficiently. In the 1980s, Gu et al. invented contralateral C7 nerve root transfer. They anastomosed the contralateral C7 nerve root with the ulnar nerve of the affected limb, so that the affected upper limb could be connected with the ipsilateral cerebral cortex (14).

Furthermore, Zheng et al. reported that upper limb motor function in patients with unilateral arm paralysis could be improved by anastomosing the contralateral C7 nerve root with that of the paralyzed side (15). Later, it was found that contralateral $\mathrm{C} 7$ nerve root transfer could induce the cerebral cortex to undergo reorganization, leading to a single hemisphere simultaneously controlling both the upper limbs $(16,17)$. This approach has the potential to strengthen the ipsilateral cortex to control the affected limb after CNSI. Hence, we speculated whether the contralateral lumbosacral nerve root could be transferred and anastomosed with the nerve root innervating the affected limb to establish a new neural pathway. In our previous study in rhesus monkeys, we found that severing the lumbosacral 6 (L6) nerve root [equivalent to the sacral 1 (S1) nerve root in humans] had no marked effect on the motor function of the ipsilateral lower limbs. This indicated that the S1 nerve root could be a source for nerve transfer to repair lower extremity nerve injury (18). When we used the normal lumbosacral nerve root for the recovery of bladder urination function, we found that after severing the S1 nerve root, patients' subjective feelings of crus muscle strength decreased slightly. However, after 3 months, muscle strength returned to normal. This showed that cutting off a single S1 nerve root has no marked effect on the motor function of the lower limbs (19). In addition, our previous animal experimental study confirmed that the transfer of the contralateral L4 nerve root to the L5 nerve root of the paralyzed side could markedly reduce spasticity and improve the motor function of the paralyzed limb (20).

Therefore, based on previous studies, we plan to develop a new treatment method to recover paralyzed limb motor function by transferring the contralateral S1 nerve root to the distal end of the severed S1 nerve root of the affected side. Notably, the motor function network of patients will be assessed by resting-state functional MRI (rs-fMRI) after the intervention to explore the detailed mechanistic changes in the brain (21). Blood oxygen level-dependent fMRI (BOLD-fMRI) is used to observe brain activity based on hemodynamic changes in different functional brain areas. When a part of the brain becomes active, the brain blood flow of the activation area increases, leading to increases in the blood oxygen level and the relative proportion of oxygenated hemoglobin and deoxyhemoglobin. Then, the compliance of the activation area changes, resulting in the MRI signal variations. Rs-fMRI is a mode of BOLD-fMRI. Unlike task-state fMRI requiring subjects to complete the task, subjects just need to stay awake with their eyes closed, breathe gently, minimize active and passive movements, and avoid thinking (22). This study has been planned to provide 
a comprehensive and effective treatment regimen for paralyzed patients in the chronic phase of CNSI. Herein, we present the study protocol following the SPIRIT reporting checklist (available at http://dx.doi.org/10.21037/ apm-21-183).

\section{Methods}

\section{Study design}

A randomized controlled clinical trial will be conducted at Shanghai General Hospital (Shanghai, China) to verify the effectiveness of contralateral S1 nerve root transfer to reestablish motor function of the lower limb in hemiplegic patients after CNSI. A total of 40 patients in the chronic phase of CNSI with lower-limb paralysis will be randomly divided into the experimental and control groups. Shanghai General Hospital will be responsible for recruiting, screening, intervention, and follow-up analysis of all participants and will assess all the outcomes and data.

The experimental group will undergo the designed S1 nerve root transfer surgery and rehabilitation. The control group will only undergo rehabilitation without the surgery. The results will be evaluated by comparing the indices of multiple tests, in which the primary criterion will be the Fugl-Meyer Assessment scale score, assessed at specific time points. Moreover, rs-fMRI will be employed to observe the variation of the connection within the motor function network after surgery and to explore the latent mechanism of motor function restoration in the brain.

This protocol was developed based on the Standard Protocol Items: Recommendations for Interventional Trials (SPIRIT) 2013 statement $(23,24)$. The study will be conducted following the Declaration of Helsinki (as revised in 2013), and the trial was approved by the Shanghai General Hospital Institutional Review Board (No. [2020]81). Participants or their legal guardians will be required to provide written informed consent. The participant flowchart is shown in Figure 1, and details of the study schedule, interventions, and assessment are given in Table 1.

\section{Study participants and inclusion and exclusion criteria}

Only eligible patients who visit Shanghai General Hospital and cooperative hospitals for treatment and are willing to undergo surgery will be recruited into this program. Participants who meet the following criteria will be included:
(I) unilateral lower-limb paralysis due to any cause (e.g., stroke, TBI, or cerebral palsy) that leads to apparent motor dysfunction and walking disability, such as obvious strephenopodia resulting in the foot being unable to reach the ground while walking and the need for orthosis, excessive flexion reflex occurring when the paralyzed side foot touches the ground leading to standing disability, and walking with the lower limb dragging because of hip joint disability for flexion; (II) no severe cognitive impairment, no sensory aphasia, and the ability to understand basic commands; (III) age between 15 and 60 years with an onset time of $\geq 12$ months; (IV) dissatisfactory functional recovery of the lower extremity after 6 months of standard rehabilitation, with eventual cessation of recovery; $(\mathrm{V})$ adult patients or parents of minor patients with the willingness to improve the hemiplegic limb and voluntarily participate in this program and agree with and sign the consent form; and (VI) MRI confirmation of only the contralateral cerebral hemisphere of the affected limb being damaged and the other side being normal.

Participants who meet the following criteria will be excluded: (I) history of chronic bone and joint diseases or joint replacement; (II) presence of diabetes mellitus, severe systemic disease of the heart, lung, or kidney, and intolerance for surgery; (III) ankylosis as the main characteristic and with severe fixed contraction or joint abnormality; (IV) inability to maintain balance; $(\mathrm{V})$ requirement of termination of the surgery for personal and/or family reasons; and (VI) severe cognitive deficits or psychological and mental disorders. Patients who meet the relative exclusion criteria will be judged on the rationality of whether to participate by at least 2 authors. The relative exclusion criteria will be hypophrenia or reduced ability to communicate.

\section{Exit criteria}

The exit criteria are as follows: (I) loss to follow-up; (II) poor compliance and disobedience of medical instructions; (III) voluntary quitting; (IV) death from any cause before the end of the program; (V) being found unqualified for the program after the initial enrollment; and (VI) severe somatopathy after the initial enrollment.

\section{Sample size}

Based on our preliminary study, we estimated that a sample size of 32 ( $\mathrm{n}=16$ per group), under the assumption of a $20 \%$ dropout rate, would provide $90 \%$ power to detect a mean $( \pm \mathrm{SD})$ intergroup difference of $8.0 \pm 6.0$ 


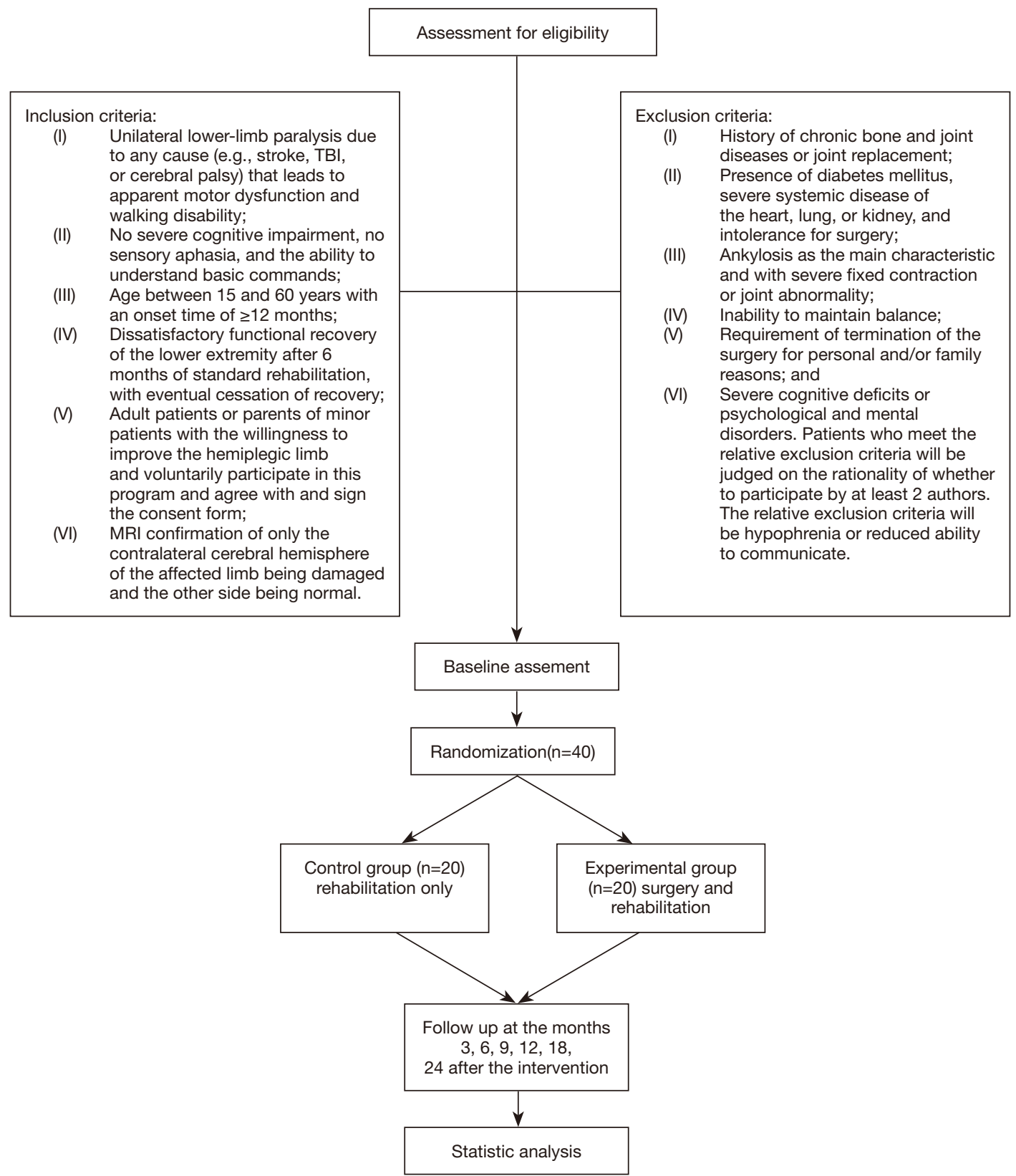

Figure 1 Flow diagram of the participants.

on the Fugl-Meyer scale. The sample sizes for each group were calculated according to the following formula, with a type I error of $5 \%(\alpha=0.05)$ and type II error of $10 \%(\beta=0.1)$ :

$$
\mathrm{n}_{1}=n_{2}=2\left[\frac{\left(u_{\alpha}+u_{\beta}\right)}{\delta / \sigma}\right]^{2}+\frac{1}{4} u_{\alpha}^{2}
$$

For practicality, 40 patients ( $\mathrm{n}=20$ per group) will be screened and enrolled in the trial.

\section{Randomization and blinding}

Based on a similar previous study (15), patients will be randomly divided into surgery and control groups using a sealed envelope, with 20 participants in each group and 40 participants in total. The randomization sequences will 
Table 1 Schedule of enrollment, interventions, and assessment

\begin{tabular}{|c|c|c|c|c|c|c|c|c|c|c|c|}
\hline \multirow{4}{*}{ Time point } & \multicolumn{11}{|c|}{ Time period } \\
\hline & \multirow{3}{*}{$\begin{array}{l}\text { Enrollment } \\
\text { ( } 2 \text { weeks before } \\
\text { intervention) }\end{array}$} & \multirow{3}{*}{$\begin{array}{l}\text { Grouping } \\
\text { (1 week before } \\
\text { intervention) }\end{array}$} & \multirow{3}{*}{ Surgery } & \multicolumn{8}{|c|}{ Post-grouping } \\
\hline & & & & \multirow{2}{*}{$\begin{array}{l}\text { Recovery after } \\
\text { surgery }\end{array}$} & \multicolumn{7}{|c|}{ Rehabilitation procedure (months) } \\
\hline & & & & & 0 & 3 & 6 & 9 & 12 & 18 & 24 (close out) \\
\hline \multicolumn{12}{|l|}{ Enrollment } \\
\hline Informed consent & • & & & & & & & & & & \\
\hline Randomization & $\bullet$ & & & & & & & & & & \\
\hline Grouping & & $\bullet$ & & & & & & & & & \\
\hline \multicolumn{12}{|l|}{ Intervention } \\
\hline \multicolumn{12}{|c|}{ Measurement and assessment } \\
\hline $\begin{array}{l}\text { Simplified Fugl-Meyer } \\
\text { assessment scale }\end{array}$ & & $\bullet$ & & & & $\bullet$ & $\bullet$ & $\bullet$ & $\bullet$ & $\bullet$ & $\bullet$ \\
\hline Modified Ashworth scale & & $\bullet$ & & & & $\bullet$ & $\bullet$ & $\bullet$ & $\bullet$ & $\bullet$ & $\bullet$ \\
\hline Modified Barthel scale & & $\bullet$ & & & & $\bullet$ & $\bullet$ & $\bullet$ & $\bullet$ & $\bullet$ & - \\
\hline $10-\mathrm{m}$ walk test & & $\bullet$ & & & & $\bullet$ & $\bullet$ & $\bullet$ & $\bullet$ & $\bullet$ & $\bullet$ \\
\hline MRC muscle force scale & & $\bullet$ & & & & $\bullet$ & $\bullet$ & $\bullet$ & $\bullet$ & $\bullet$ & $\bullet$ \\
\hline $3 \mathrm{D}$ gait analysis & & $\bullet$ & & & & $\bullet$ & $\bullet$ & $\bullet$ & $\bullet$ & $\bullet$ & - \\
\hline
\end{tabular}

According to the SPIRIT 2013 statement: defining standard protocol items for clinical trials. MRC, the UK Medical Research Council.

be computer-generated by an independent statistician and will remain unknown to trial personnel until assignment. The results analysts will not be involved in the allocation and interventions and will be blinded to the allocation until statistical analyses are completed.

\section{Interventions}

Based on our previous work, we suggested that S1 nerve anastomosis from the healthy side to the paralyzed side would improve the motor ability of the paralyzed limb. Study participants will be divided into control and experimental groups. The primary demographic and clinical information, motor index, living ability, and psychological condition of both groups will be evaluated before their respective therapies.

The control group (Group A) will undergo standard rehabilitation training, including rolling up, maintaining sitting or standing balance, sitting-stand switching, weight transition, unilateral limb weight training, and walking training.

The experimental group (Group B) will undergo the proposed surgery of transferring the contralateral S1 nerve root to the distal end of the severed S1 nerve root of the affected side (Figure 2). Patients in this group will undergo the same rehabilitation training as those in the control group. Any type of rehabilitation therapy that is not included in the protocol will be prohibited. 


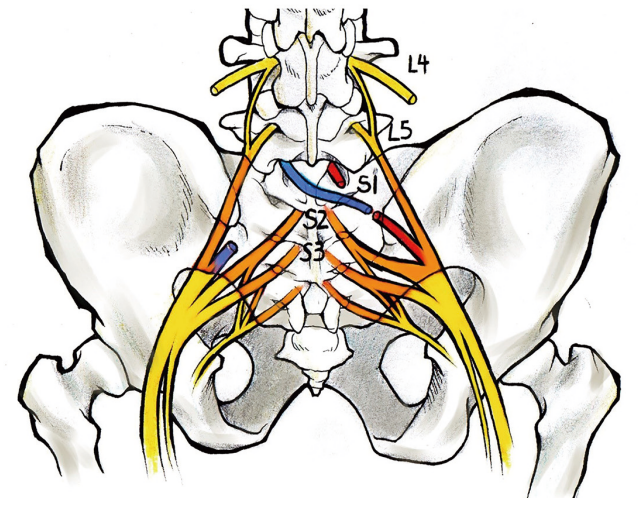

Figure 2 Illustration of the contralateral S1 nerve root transfer surgery.

\section{Detailed surgery design}

The surgery will be performed with the patients in the prone position after induction of general anesthesia. The surgical area will be disinfected and draped. Taking the spinous process of L5 as the center, a middle, longitudinal, $10-\mathrm{cm}$ long incision will be made. The skin and tissue under the skin and fascia layer will be cut open successively. The musculus sacrospinalis will be stripped along the L4, L5, and S1 spinous processes, and the vertebral plates and facet joints will be uncovered. After eliminating the residual muscle tissue, the skin and the soft tissue will be separated with automatic retractors. After confirming the nerve, the L5 spinous process will be excised, along with the L5 vertebral plates and ligamentum flavum by total laminectomy. Part of the sacrum will be excised downward, and the dural sac will be fully uncovered. The endorachis and the arachnoid membrane will be cut open, and the cauda equina nerve will be separated with a nerve stripper and retractors. After identifying the S1 nerve root, it will be stimulated by an electrophysiological stimulus. The ankle of the same side should contract strongly if the S1 nerve functions properly. Then, the S1 nerve root on the paralyzed side will be exposed. The S1 nerve root of both sides will be excised, and the proximal end of the S1 nerve root will be transferred and anastomosed to the distal end of the $\mathrm{S} 1$ nerve on the paralyzed side. Then, the dural sac and incision will be closed layer-by-layer.

\section{Efficacy evaluation criteria}

The Fugl-Meyer assessment scale, the Modified Ashworth scale, the Modified Barthel scale, 3D gait analysis, muscle force evaluations, the 10-m walk test, electromyography (EMG), and rs-fMRI will be used to evaluate the efficacy of the treatment for each patient in the program by 1 member in the research group before and 3, 6, 9, 12, 18, and 24 months after the intervention.

\section{Primary endpoint criteria}

The Fugl-Meyer assessment scale index at 24 months after treatment, which is aimed at assessing stroke patients, includes evaluations of motor ability, sensation, balance, range of motion of joints, and pain. Among these, motor ability covers 17 categories and 50 items. Each item accounts for 2 points, and the total score for the lower limbs is 34 points. We will employ the Fugl-Meyer assessment scale to evaluate patients' lower limb function recovery in this study.

\section{Secondary endpoint criteria}

Assessment of muscular tone will be performed by the Modified Ashworth scale, while the modified Barthel scale will measure activities of daily living. The $10-\mathrm{m}$ walk test will be used to measure the maximal and mean speed of a $10-\mathrm{m}$ walk under comfortable conditions. Muscle force will be evaluated according to the UK Medical Research Council (MRC) muscle force scale amended in 1999.

A 3D gait analysis will quantify the time-space parameter and kinematic parameter, including the spatial angle of the hip joint, walk speed, footstep frequency, footstep length, footstep width, time of support by the unilateral lower limb, and the motor trajectory, and compare gait before and after treatment to precisely assess the walkability of the lower limb.

EMG will be measured by determining the myoelectric potential/evoked potential meter. The device will collect the following information: (I) the velocity of sensation or motor nerve conduction; (II) the amplitude, duration of the insertion potential, abnormal spontaneous potential, the potential of the motor unit; (III) the raising potential when the muscle contracts. EMG aims to evaluate the reinnervation of significant muscles of the lower limb.

Rs-fMRI will be conducted with the Siemens 3.0 T Trio TimSystem with a static function scan (relevant parameters: $\mathrm{TR}=2,000 \mathrm{~ms}, \mathrm{TE}=30 \mathrm{~ms}$, flip angle $=900$, slices $=31$, resolution $=64 \times 64$ ) and the T1-MPRAG sequence (relevant parameters: $\mathrm{TR}=1,900 \mathrm{~ms}$, $\mathrm{TE}=213 \mathrm{~ms}$, $\mathrm{TI}=900 \mathrm{~ms}$, flip angle $=900$, slices $=176$, resolution $=256 \times 256$ ). The participants will lie down on the scanning bed with their heads fixed properly. They will wear earplugs to reduce the noise level when inside the scanner. Patients will be 
instructed to relax, breathe gently with eyes closed, maintain their body as still as possible, and try not to engage in mental activities. The changes in the motor functioning network will be observed and recorded before and after the treatment.

\section{Safety outcomes}

Safety outcomes include adverse events and changes in the motor and sensory function of the limb on the side of the severed donor S1 nerve over 12 months. The changes will be assessed by evaluating muscle strength, tactile sensory threshold, two-point discrimination, and electrophysiological findings.

\section{Assessment of adverse events}

Adverse events that can be observed will be recorded using a specific chart. The following question will be used to collect information directly regarding adverse events: "Do you feel anything different since the last examination?" Every bar in the chart represents 1 adverse event. The patients should sign their names below the chart regardless of whether they experience adverse events. If any adverse event occurs, the researcher will provide the corresponding treatment to the patient. The adverse events will be immediately reported to the primary investigator to determine if the patient needs to withdraw from the trial.

\section{Data collection and management}

Baseline characteristic data will be collected by screeners when screening potential participants before the study, and the essential parameters will be continuously monitored during the trial. The Fugl-Meyer assessment scale, the Modified Ashworth scale, the Modified Barthel scale, 3D gait analysis, muscle force, the 10-m walk test, EMG, and rsfMRI will be used to evaluate the efficacy of the treatment for each patient in the program by 1 member of the research group before and at 3, 6, 9, 12, 18, and 24 months after the treatment. The researchers will maintain the quality of data collection and be responsible for data entry. The data administrator will be responsible for identifying, encoding, and processing the initial data for data analysis.

\section{Statistical analysis}

All analyses will be based on the intention-to-treat principle. Any missing value will be filled in with the last observation carried forward method. The data will be statistically processed by the Statistical Department of Shanghai Jiao Tong University School of Medicine with SPSS 23.0 statistical software. The quantitative data will be expressed as mean \pm standard deviation $(\mathrm{SD})$, and the minimum, maximum, and median values will be reported. For enumerative variables, the frequency and corresponding percentage will be assigned. For continuous variables, the $t$-test (or Satterthwaite's method) or Wilcoxon rank-sum tests will be used for inter-group comparisons. Paired $t$-tests, Wilcoxon matched-pairs signed-ranks tests, and repeated ANOVA will be used for intra-group comparisons between each follow-up visit and baseline. For discrete variables, chisquare or Fisher's exact tests will be used for inter-group comparisons, and McNemar's chi-square tests will be used for intra-group comparisons. Sensitivity analysis will be performed by using the $\mathrm{PP}$ subset. Two-tailed $\mathrm{P}<0.05$ will be considered to indicate statistically significant differences.

\section{Discussion}

CNSI is one of the leading causes of paralysis. The global prevalence of stroke was 104.2 million in 2017 (25). Every year, approximately 795,000 people experience a new or recurrent stroke in the US, and approximately $3 \%$ of male and $2 \%$ of female patients report stroke-related disabilities (25). Worldwide, more than 50 million people experience TBI each year (26). The present treatments focus on the acute phase of CNSI. Thus, for paralyzed patients in the chronic phase, there is a serious lack of effective treatments.

Spontaneous recovery of limb dysfunction after stroke is mainly achieved within 3 months after stroke onset. However, after 3 months, the process of recovery enters the chronic phase, and the ability and progress of spontaneous recovery become extremely slow (27). At present, rehabilitation training is the main treatment for the chronic stage of hemiplegia after stroke, but only approximately $30 \%$ of patients can partly recover motor function, and the outcomes of this treatment are poor (28). Previous studies have identified inhibition and facilitation areas in the reticular formation of the brainstem; the inhibition area causes relaxation of the limbs, while the facilitation area makes the limbs tense (29). Under normal physiological conditions, both areas are in balance. However, in patients with stroke, cerebral palsy, and other CNSIs, damage of the motor neurons in the cerebral cortex causes patients to lose the initiating effect on the inhibition area, enhances the relative effect of the facilitation area, and increases the 
muscle tension of the limbs dominated by them, resulting in a stiff state (30). For patients with cerebral palsy, selective dorsal rhizotomy is often used in clinical treatment, which can effectively relieve limb spasms by selectively cutting the dorsal root of the spinal nerve, thus creating good conditions for further limb rehabilitation training (31). Therefore, we believe that contralateral lumbosacral nerve root transfer can be used to anastomose with the affected lumbosacral nerve root to reconstruct limb motor function in hemiplegic patients. The affected spinal nerve can be excised for anastomosis during the operation, which is similar to SPR. This method can relax the spastic paralyzed limb by cutting off the lower nerve reflex loop of the spastic limb and achieving the goal of nerve regeneration through nerve transfer to reinnervate the related muscles of the affected lower limbs. In combination with postoperative rehabilitation training, this procedure is expected to improve the degree of limb function recovery.

In summary, based on previous studies, we designed this single-center randomized controlled trial to verify the effect of contralateral S1 nerve root transfer to reconstruct the motor function of paralyzed lower limbs. We aim to use generally accepted scales as our outcomes and rs-fMRI for further exploration of brain reorganization. We believe that our study will provide a comprehensive and effective treatment regimen for the motor function reconstruction of paralyzed limbs after CNSI.

\section{Acknowledgments}

Funding: This work was supported by the Clinical Research Plan of SHDC (grant number: SHDC2020CR4024), the Medical Innovation Research Project of Shanghai Science and Technology Commission (grant number: 20Y11913100), and the Research Physician Project of Shanghai Jiao Tong University School of Medicine.

\section{Footnote}

Reporting Checklist: The authors have completed the SPIRIT reporting checklist. Available at http://dx.doi.org/10.21037/ apm-21-183

Conflicts of Interest: All authors have completed the ICMJE uniform disclosure form (available at http://dx.doi. org/10.21037/apm-21-183). The authors have no conflicts of interest to declare.
Ethical Statement: The authors are accountable for all aspects of the work in ensuring that questions related to the accuracy or integrity of any part of the work are appropriately investigated and resolved. The study will be conducted following the Declaration of Helsinki (as revised in 2013), and the trial was approved by the Shanghai General Hospital Institutional Review Board (No. [2020]81). Participants or their legal guardians will be required to provide written informed consent.

Open Access Statement: This is an Open Access article distributed in accordance with the Creative Commons Attribution-NonCommercial-NoDerivs 4.0 International License (CC BY-NC-ND 4.0), which permits the noncommercial replication and distribution of the article with the strict proviso that no changes or edits are made and the original work is properly cited (including links to both the formal publication through the relevant DOI and the license). See: https://creativecommons.org/licenses/by-nc-nd/4.0/.

\section{References}

1. Sauser K, Burke JF, Reeves MJ, . A systematic review and critical appraisal of quality measures for the emergency care of acute ischemic stroke. Ann Emerg Med 2014;64:235-44.e5.

2. Lo AC, Guarino PD, Richards LG, et al. Robot-assisted therapy for long-term upper-limb impairment after stroke. $\mathrm{N}$ Engl J Med 2010;362:1772-83.

3. Thieme H, Morkisch N, Mehrholz J, et al. Mirror therapy for improving motor function after stroke. Cochrane Database Syst Rev 2018;7:CD008449.

4. Sun P, Liu DZ, Jickling GC, et al. MicroRNA-based therapeutics in central nervous system injuries. J Cereb Blood Flow Metab 2018;38:1125-48.

5. O’Donnell MJ, Xavier D, Liu L, et al. Risk factors for ischaemic and intracerebral haemorrhagic stroke in 22 countries (the INTERSTROKE study): a case-control study. Lancet 2010;376:112-23.

6. Lambert C, Cisternas P, Inestrosa N. Role of Wnt Signaling in Central Nervous System Injury. Mol Neurobiol 2016;53:2297-311.

7. Strong K, Mathers C, Bonita R. Preventing stroke: saving lives around the world. Lancet Neurol 2007;6:182-7.

8. Edvinsson LI, Povlsen G. Vascular plasticity in cerebrovascular disorders. J Cereb Blood Flow Metab 2011;31:1554-71.

9. Steinle B, Corbaley J. Rehabilitation of stroke: a new horizon. Mo Med 2011;108:284-8.

10. Mir O, Savitz S. Stem cell therapy in stroke treatment: is it a 
viable option? Expert Rev Neurother 2013;13:119-21.

11. Lee DH, Lee JY, Oh BM, et al. Functional recovery after injury of motor cortex in rats: effects of rehabilitation and stem cell transplantation in a traumatic brain injury model of cortical resection. Childs Nerv Syst 2013;29:403-11.

12. Jankowska E, Edgley SA. How can corticospinal tract neurons contribute to ipsilateral movements? A question with implications for recovery of motor functions. Neuroscientist 2006;12:67-79.

13. Ward NS, Brown M, Thompson A, et al. Neural correlates of outcome after stroke: a cross-sectional fMRI study. Brain 2003;126:1430-48.

14. Gu YD, Chen D, Zhang G, et al. Long-term functional results of contralateral C7 transfer. Journal of reconstructive microsurgery 1998;14:57-9.

15. Zheng MX, Hua XY, Feng JT, et al. Trial of Contralateral Seventh Cervical Nerve Transfer for Spastic Arm Paralysis. New Engl J Med 2018;378:22-34.

16. Jiang S, Li Z, Hua X, et al. Reorganization in motor cortex after brachial plexus avulsion injury and repair with the contralateral C7 root transfer in rats. Microsurgery 2010;30:314-20.

17. Harris NG, Chen S, Pickard J. Cortical reorganization after experimental traumatic brain injury: a functional autoradiography study. J Neurotrauma 2013;30:1137-46.

18. Lin H, Xu Z, Liu Y, et al. The effect of severing L6 nerve root of the sacral plexus on lower extremity function: an experimental study in rhesus monkeys. Neurosurgery 2012;70:170-7; discussion 7.

19. Lin H, Hou C. Transfer of normal S1 nerve root to reinnervate atonic bladder due to conus medullaris injury. Muscle Nerve 2013;47:241-5.

20. Zong H, Ma F, Zhang L, et al. Hindlimb spasticity after unilateral motor cortex lesion in rats is reduced by contralateral nerve root transfer. Biosci Rep 2016;36:e0430.
21. Bigler ED. Traumatic brain injury, neuroimaging, and neurodegeneration. Front Hum Neurosci 2013;7:395.

22. Wang R, Liu N, Tao YY, et al. The Application of rs-fMRI in Vascular Cognitive Impairment. Front Neurol 2020;11:951.

23. Chan AW, Tetzlaff JM, Gøtzsche PC, et al. SPIRIT 2013 explanation and elaboration: guidance for protocols of clinical trials. BMJ 2013;346:e7586.

24. Chan AW, Tetzlaff JM, Altman DG, et al. SPIRIT 2013 statement: defining standard protocol items for clinical trials. Ann Intern Med 2013;158:200-7.

25. Virani SS, Alonso A, Benjamin E, et al. Heart Disease and Stroke Statistics-2020 Update: A Report From the American Heart Association. Circulation 2020;141:e139-e596.

26. Jiang JY, Gao G, Feng J, et al. Traumatic brain injury in China. Lancet Neurol 2019;18:286-95.

27. Heitsch LE, Panagos PD. Treating the elderly stroke patient: complications, controversies, and best care metrics. Clin Geriatr Med 2013;29:231-55.

28. Romero JR, Babikian VL, Katz DI, et al. Neuroprotection and stroke rehabilitation: modulation and enhancement of recovery. Behav Neurol 2006;17:17-24.

29. Moschovakis AK, Kitama T, Dalezios Y, et al. An anatomical substrate for the spatiotemporal transformation. J Neurosci 1998;18:10219-29.

30. Arpin DJ, Stuberg W, Stergiou N, et al. Motor control of the lower extremity musculature in children with cerebral palsy Res Dev Disabil 2013;34:1134-43.

31. Langerak NG, Hillier SL, Verkoeijen PP, et al. Level of activity and participation in adults with spastic diplegia 1726 years after selective dorsal rhizotomy. J Rehabil Med 2011;43:330-7.

(English Language Editors: C. Betlazar-Maseh and J. Chapnick)
Cite this article as: Peng Y, Kan SF, Ren WJ, Li ZX, Yin G, $\mathrm{Yu}$ B, Lin HD. Contralateral S1 nerve root transfer for motor function recovery in the lower extremity among patients with central nervous system injury: study protocol for a randomized controlled trial. Ann Palliat Med 2021;10(6):6900-6908. doi: 10.21037/apm-21-183 